\title{
Domain and parameter reconstruction in photothermal imaging
}

Ana Carpio and María-Luisa Rapún

\begin{abstract}
In this work we address the inverse problem of reconstructing inclusions and their thermal parameters given temperature measurements at the accessible side of a material. We describe an iterative descent method that combines topological derivative computations to reconstruct the geometry of the defects with gradient iterations to approximate the material parameters. A numerical experiment showing the ability of the method to obtain reasonable reconstructions in a few iterations is presented.
\end{abstract}

\section{Statement of the problem}

Photothermal imaging techniques are suitable means of inspecting composite materials with nondestructive tests. In this work we develop techniques to detect defects $\Omega$ buried in a medium by surface thermal measurements. We are interested in a photothermal technique that consists in heating the surface of a semi-infinite medium by a laser beam and recording the temperature at several receptors located on the same surface during a time interval, see Fig. 1. Recent physical experiments using this kind of technique can be found in $[9,18]$.

The forward problem is modelled by a heat diffusion equation in the half plane $\mathbb{R}_{-}^{2}:=\left\{(x, y) \in \mathbb{R}^{2}, \quad y<0\right\}$. The surface of the sample $\Pi:=\{(x, 0), \quad x \in \mathbb{R}\}$ is thermically excited with a delta-pulse located at a source point $\mathbf{x}_{0} \in \Pi$, generating a thermal wave of the form

Ana Carpio

Dep. Matemática Aplicada, Fac. Matemáticas, Universidad Complutense de Madrid, 28040

Madrid, Spain e-mail: ana_carpio@mat.ucm.es

María-Luisa Rapún

Dep. Fundamentos Matemáticos, ETSI Aeronáuticos, Universidad Politécnica de Madrid, 28040

Madrid, Spain e-mail: marialuisa.rapun@upm.es 


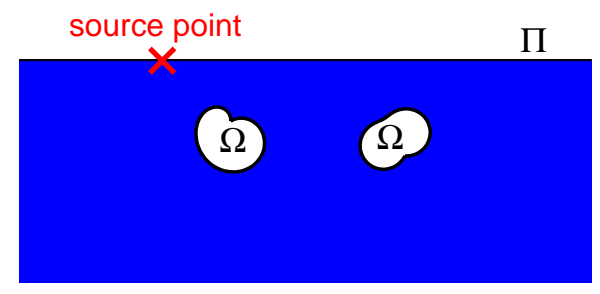

Fig. 1 Geometrical configuration. The source and observation points are located on the boundary $\Pi$ of the medium. The objects $\Omega$ and their physical parameters are the unknowns of the phothermal imaging problem.

$$
U_{\text {inc }}(\mathbf{x}, t)=(1 / t) \exp \left(-\rho_{e}\left|\mathbf{x}-\mathbf{x}_{0}\right|^{2} /\left(4 \kappa_{e} t\right)\right), \quad \mathbf{x} \in \mathbb{R}^{2}, t>0
$$

Here $\kappa_{e}$ is the thermal conductivity of the exterior medium $\Omega_{e}:=\mathbb{R}_{-}^{2} \backslash \Omega$ and $\rho_{e}$ is the density multiplied by its specific heat. The corresponding thermal parameters inside the inclusions $\Omega$ are $\kappa_{i}$ and $\rho_{i}$. The temperature distribution

$$
U(\mathbf{x}, t):= \begin{cases}U_{+}(\mathbf{x}, t), & \text { in } \Omega_{e} \times(0, \infty) \\ U_{-}(\mathbf{x}, t), & \text { in } \Omega \times(0, \infty)\end{cases}
$$

satisfies the heat equations

$$
\rho_{e} \partial_{t} U_{+}=\kappa_{e} \Delta U_{+}, \quad \text { in } \Omega_{e} \times(0, \infty), \quad \rho_{i} \partial_{t} U_{-}=\kappa_{i} \Delta U_{-}, \quad \text { in } \Omega \times(0, \infty) .
$$

In the exterior domain $\Omega_{e}$, the total temperature $U_{\text {total }}=U_{+}+U_{\text {inc }}$ is the superposition of $U_{+}$and the incident wave defined in (1). The temperature satisfies the following transmission conditions at the common interface:

$$
U_{-}-U_{+}=U_{i n c}, \quad \kappa_{i} \partial_{\mathbf{n}} U_{-}-\kappa_{e} \partial_{\mathbf{n}} U_{+}=\kappa_{e} \partial_{\mathbf{n}} U_{\text {inc }}, \quad \text { on } \partial \Omega \times(0, \infty) .
$$

The forward problem is completed imposing an adiabatic boundary condition on the upper boundary $\Pi$, and homogeneous initial conditions:

$$
\partial_{\mathbf{n}} U_{+}=0, \quad \text { on } \Pi \times(0, \infty), \quad U_{+}(\mathbf{x}, 0)=U_{-}(\mathbf{x}, 0)=0, \quad \forall \mathbf{x} \in \mathbb{R}_{-}^{2} .
$$

The solution $U$ of the forward problem can be numerically approximated using the following strategy $[12,14]$ : if we consider the Laplace transform of $U$ and $U_{\text {inc }}$, $u(\mathbf{x}, s)=\int_{0}^{\infty} e^{-s t} U(\mathbf{x}, t) d t$ and $u_{i n c, s}(\mathbf{x})=\int_{0}^{\infty} e^{-s t} U_{i n c}(\mathbf{x}, t) d t$, then, for each value of $s$ the function $u_{s}(\mathbf{x}):=u(\mathbf{x}, s)$ is a radiating solution of the stationary problem 


$$
\begin{cases}\kappa_{e} \Delta u_{s}-s \rho_{e} u_{s}=0, \quad \text { in } \Omega_{e}, & \kappa_{i} \Delta u_{s}-s \rho_{i} u_{s}=0, \quad \text { in } \Omega, \\ u_{s}^{-}-u_{s}^{+}=u_{\text {inc }, s}, & \text { on } \partial \Omega \\ \partial_{\mathbf{n}} u_{s}=0, \quad \text { on } \Pi . & \kappa_{i} \partial_{\mathbf{n}} u_{s}^{-}-\kappa_{e} \partial_{\mathbf{n}} u_{s}^{+}=\kappa_{e} \partial_{\mathbf{n}} u_{i n c, s}, \quad \text { on } \partial \Omega\end{cases}
$$

To invert the Laplace transform we choose the hyperbolic paths of the form [17]: $\gamma(\theta):=\mu(1-\sin (\pi / 4+\imath \theta)), \quad \theta \in \mathbb{R}$, where $\mu>0$. Then, the solution of (2-4) is

$$
U(\mathbf{x}, t)=\frac{1}{2 \pi_{l}} \int_{-\infty}^{\infty} e^{t \gamma(\theta)} u(\mathbf{x}, \gamma(\theta)) \gamma^{\prime}(\theta) d \theta
$$

Numerical approximations of $U$ can be calculated using a truncated trapezoidal rule

$$
U(\mathbf{x}, t) \approx \sum_{\ell=-L}^{L} c_{\ell} e^{t s_{\ell}} u\left(\mathbf{x}, s_{\ell}\right)
$$

$s_{\ell}=\gamma\left(\frac{\log (L)}{L} \ell\right)$ and weights $c_{\ell}=\frac{\log (L)}{2 \pi L L} \gamma^{\prime}\left(\frac{\log (L)}{L} \ell\right)$.

The inverse problem consists in finding the objects $\Omega$ and the parameters $\kappa_{i}, \rho_{i}$ such that the solution of the forward transmission problem (2-4) equals the measured values of the total wave $U_{\text {meas }}\left(\mathbf{x}_{i}, t_{j}\right)$ at the detector locations $\mathbf{x}_{1}, \ldots, \mathbf{x}_{M} \in \Pi$ at the time instants $t_{1}, \ldots, t_{N}$. Since this problem is ill-posed, we consider a weaker variational reformulation: find $\Omega, \kappa_{i}, \rho_{i}$ minimizing the functional

$$
J\left(\mathbb{R}_{-}^{2} \backslash \Omega, \kappa_{i}, \rho_{i}\right)=\frac{1}{2} \sum_{i=1}^{M} \sum_{j=1}^{N} f\left(t_{j}\right)\left(U_{\text {total }}\left(\mathbf{x}_{i}, t_{j}\right)-U_{\text {meas }}\left(\mathbf{x}_{i}, t_{j}\right)\right)^{2},
$$

where $U_{\text {total }}$ is the solution of the forward problem (2-4) when the object is $\Omega$ and the interior thermal parameters are $\kappa_{i}$ and $\rho_{i}$. The weight function $f(t)$ normalizes the time decay of the solutions of the heat equation. For our numerical experiment in section 3 we select $f(t)=\max _{\mathbf{x} \in\left\{\mathbf{x}_{1}, \ldots, \mathbf{x}_{M}\right\}}\left|U_{\text {meas }}(\mathbf{x}, t)\right|^{-1}$. Other possibilities were explored in $[4,6]$.

Based on the Laplace-transform strategy described above, we proposed in $[4,6]$ to substitute the cost functional (6) by the approximated funcional

$$
J\left(\mathbb{R}_{-}^{2} \backslash \Omega, \kappa_{i}, \rho_{i}\right)=\frac{1}{2} \sum_{i=1}^{M} \sum_{j=1}^{N} f\left(t_{j}\right)\left(\sum_{\ell=-L}^{L} c_{\ell} e^{t_{j} s_{\ell}} u\left(\mathbf{x}_{i}, s_{\ell}\right)-U_{\text {meas }}\left(\mathbf{x}_{i}, t_{j}\right)\right)^{2},
$$

having now $2 L+1$ stationary constraints.

\section{Iterative method to reconstruct inclusions and parameters}

To solve the optimization problem we combine gradient and topological derivative (TD in the sequel) methods to generate sequences of parameters and objects in such 
a way that the cost functional decreases throughout the iterative procedure. Our choice of a TD strategy is based on the following advantageous features:

- Without a priori information, the TD provides a good first guess of the number, size and location of the inclusions. This has been tested in a wide range of physical settings, including acoustics, electromagnetism, elastodynamics, electrical impedance tomography, fluorescence optical tomography, and photothermal imaging $[1,5,7,8,10,15,20]$.

- Iterative TD methods allow for topological changes during the iterations, in contrast to classical shape deformation strategies $[11,13,19]$ that require knowledge of the number of objects from the start. Using iterative TD based methods, new objects may be created in the course of the iterations, existing contours may merge and holes inside existing objects may be detected, see [2,3]. Furthermore, even if the number of inclusions is known (assumption that in most practical applications is not realistic), TD-iterative methods are a powerful alternative to these classical methods, providing accurate reconstructions at a low computational cost, as extensively checked by the authors in different contexts (see [2-6] and references therein).

- In comparison with other strategies allowing for topological changes (as i.e. level set methods $[16,21])$, the number of iterations with respect to the domain is usually much smaller.

In our previous papers $[4,6]$ we used a non-standard formulation of the photothermal problem (2-4), involving two interior parameters related with $\kappa_{e}, \kappa_{i}, \rho_{e}$, and $\rho_{i}$ with no physical meaning. In this paper we adapt the results in $[4,6]$ to deal with the reconstruction of defects and of their physical parameters $\kappa_{i}$ and $\rho_{i}$.

The TD of a shape functional $\mathscr{J}(\mathscr{R})$ is a pointwise function defined as [22]:

$$
D_{T}(\mathbf{x}, \mathscr{R})=\lim _{\varepsilon \rightarrow 0} \frac{\mathscr{J}\left(\mathscr{R} \backslash B_{\varepsilon}(\mathbf{x})\right)-\mathscr{J}(\mathscr{R})}{\pi \varepsilon^{2}}, \quad \mathbf{x} \in \mathscr{R},
$$

where $B_{\mathcal{\varepsilon}}(\mathbf{x})$ is a ball centered at $\mathbf{x}$ with radius $\varepsilon$. Then, it follows the expansion:

$$
\mathscr{J}\left(\mathscr{R} \backslash B_{\varepsilon}(\mathbf{x})\right)=\mathscr{J}(\mathscr{R})+D_{T}(\mathbf{x}, \mathscr{R}) \pi \varepsilon^{2}+o\left(\varepsilon^{2}\right), \quad \text { as } \varepsilon \rightarrow 0 .
$$

This motivates the key idea for the reconstruction technique: if we locate small objects $B_{\mathcal{E}}(\mathbf{x})$ at the points $\mathbf{x} \in \mathscr{R}$ where $D_{T}(\mathbf{x}, \mathscr{R})$ is negative, then $\mathscr{J}\left(\mathscr{R} \backslash B_{\mathcal{E}}(\mathbf{x})\right)<$ $\mathscr{J}(\mathscr{R})$, that is, the value of the functional decreases. Hence we will identify the points where the TD attains the larger negative values with the regions where it is more likely to have an object.

The next result can be proved following Theorem 3.2 in [4].

Theorem 1. The TD of the functional $J\left(\mathbb{R}_{-}^{2} \backslash \Omega, \kappa_{i}, \rho_{i}\right)$ defined in (7) is

$$
D_{T}(\mathbf{x})=\operatorname{Re}\left(\sum_{\ell=-L}^{L} \frac{2 \kappa_{e}\left(\kappa_{e}-\kappa_{i}\right)}{\kappa_{e}+\kappa_{i}} \nabla u_{t o t a l, s_{\ell}}(\mathbf{x}) \nabla p_{s_{\ell}}(\mathbf{x})+\left(\rho_{e}-\rho_{i}\right) s_{\ell} u_{t o t a l, s_{\ell}}(\mathbf{x}) p_{s_{\ell}}(\mathbf{x})\right)
$$


for $\mathbf{x} \in \mathbb{R}_{-}^{2} \backslash \Omega$, where $u_{\text {total }, s_{\ell}}=u_{\text {inc, } s_{\ell}}+u_{s_{\ell}}$ and $u_{s_{\ell}}$ is the solution of (5) for $s=s_{\ell}$. The adjoint fields $p_{s_{\ell}}$ are solutions of:

$$
\begin{cases}\kappa_{e} \Delta p_{s_{\ell}}-s_{\ell} \rho_{e} p_{s_{\ell}}=g_{s_{\ell}}, \quad \text { in } \Omega_{e}, & \kappa_{i} \Delta p_{s_{\ell}}-s_{\ell} \rho_{i} p_{s_{\ell}}=0, \quad \text { in } \Omega, \\ p_{s_{\ell}}^{-}-p_{s_{\ell}}^{+}=0, \quad \text { on } \partial \Omega & \kappa_{i} \partial_{\mathbf{n}} p_{s_{\ell}}^{-}-\kappa_{e} \partial_{\mathbf{n}} p_{s_{\ell}}^{+}=0, \quad \text { on } \partial \Omega \\ \partial_{\mathbf{n}} p_{s_{\ell}}=0, \quad \text { on } \Pi, & \end{cases}
$$

with $g_{s_{\ell}}(\mathbf{x}):=\sum_{i=1}^{M} \sum_{j=1}^{N} f\left(t_{j}\right) c_{s_{\ell}} e^{t_{j} s_{\ell}}\left(\overline{U_{\text {meas }}\left(\mathbf{x}_{i}, t_{j}\right)-\sum_{k=-L}^{L} c_{k} e^{t_{j} s_{k}} u_{s_{k}}\left(\mathbf{x}_{i}\right)}\right) \delta_{\mathbf{x}_{i}}(\mathbf{x})$.

By iteratively applying Theorem 1 , we construct a monotone sequence of approximate domains $\Omega_{d} \subset \Omega_{d+1}$ adding to the current approximation $\Omega_{d}$ the points where the TD attains pronounced negative values. To be able to remove points from $\Omega_{d}$, we need to compute the TD inside the inclusions. The definition (8) can be extended to the points inside $\Omega$ [3], and an analogous expression to (9) can be found for $\mathbf{x} \in \Omega[5,6]$. This extension is the basis to develop iterative strategies able to correct an approximation of $\Omega$ by removing the points where the TD attains pronounced positive values.

To determine the thermal parameters we proceed as follows. If $\widetilde{\kappa}_{i}, \widetilde{\rho}_{i}$ are approximate values of $\kappa_{i}$ and $\rho_{i}$, and $\widetilde{\Omega}$ is an approximation of $\Omega$, then we correct the values $\widetilde{\kappa}_{i}, \widetilde{\rho}_{i}$ by a gradient method. The idea is to define $\kappa_{i}=\widetilde{\kappa}_{i}+\eta \phi, \quad \rho_{i}=$ $\widetilde{\rho}_{i}+\eta \psi$, where $\eta>0$ is small and $\phi, \psi$ are selected calculating the derivative of $J(\eta):=J\left(\widetilde{\Omega}, \widetilde{\kappa}_{i}+\eta \phi, \widetilde{\rho}_{i}+\eta \psi\right)$ with respect to $\eta$ to ensure that $J^{\prime}(0)<0$. A procedure to obtain explicit formulae in terms of forward and adjoint fields for this kind of functionals is explained in $[3,6]$. In our case, it can be proven that the choice

$$
\phi=\operatorname{Re}\left(\int_{\widetilde{\Omega}} \sum_{\ell=-L}^{L} \nabla u_{s_{\ell}} \nabla p_{s_{\ell}}\right), \quad \psi=\operatorname{Re}\left(\int_{\widetilde{\Omega}} \sum_{\ell=-L}^{L} s_{\ell} u_{s_{\ell}} p_{s_{\ell}}\right),
$$

makes $J^{\prime}(0)<0$. Here $u_{s_{\ell}}, p_{s_{\ell}}$ are solutions of (5) and (10), respectively, with $\Omega=$ $\widetilde{\Omega}, \kappa_{i}=\widetilde{\kappa}_{i}$ and $\rho_{i}=\widetilde{\rho}_{i}$.

Finally, our procedure is as follows. In a first step we consider initial guesses of the parameters $\kappa_{i}=\kappa_{i}^{0}, \rho_{i}=\rho_{i}^{0}$ and compute the TD in $\mathbb{R}_{-}^{2}$ for these parameters, that is, the TD of $J\left(\mathbb{R}_{-}^{2}, \kappa_{i}^{0}, \rho_{i}^{0}\right)$. We find then a first approximation $\Omega_{1}$ of $\Omega$ as the union of all the points where the TD is smaller than a negative constant (see $[3,4]$ for guidelines of the selection of such constant). Once $\Omega_{1}$ is set, we update the values of the parameters performing $Q$ iterations of the gradient method ( $Q=8$ in our numerical example in Section 3) as explained above:

$$
\kappa_{i}^{q}=\kappa_{i}^{q-1}+\eta \phi^{q}, \quad \rho_{i}^{q}=\rho_{i}^{q-1}+\eta \psi^{q}, \quad q=1, \ldots, Q
$$

with $\phi^{q}, \psi^{q}$ defined as in (11) with $\widetilde{\Omega}=\Omega_{1}, \widetilde{\kappa}_{i}=\kappa_{i}^{q-1}$ and $\widetilde{\rho}_{i}=\rho_{i}^{q-1}$. Once the parameters are corrected, we compute the TD of $J\left(\mathbb{R}_{-}^{2} \backslash \Omega_{1}, \kappa_{i}^{Q}, \rho_{i}^{Q}\right)$ to update the domain $\Omega_{1}$ by adding to it the points $\mathbf{x} \in \mathbb{R}_{-}^{2} \backslash \Omega_{1}$ where the TD attains the larger negative values, and removing from $\Omega_{1}$ the points inside it, if any, where the TD attains the larger positive values. Once the approximation of the domains is im- 
(a)

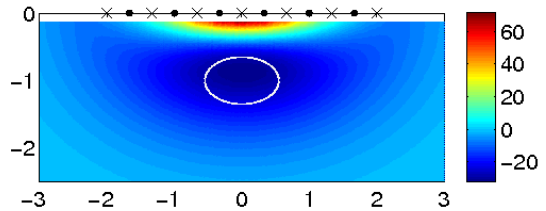

(c)

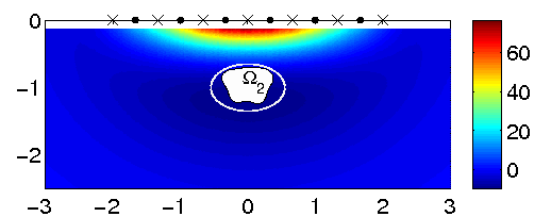

(b)

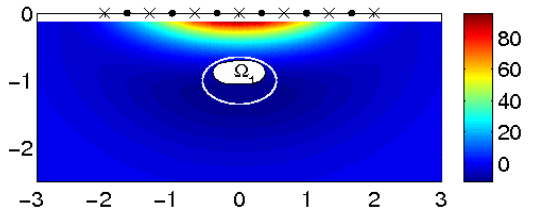

(d)

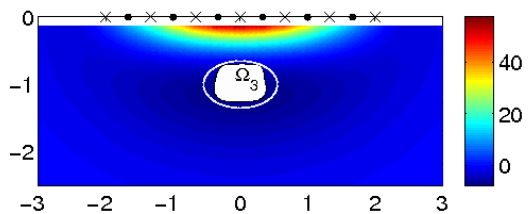

Fig. 2 (a) Topological derivative when $\kappa_{i}^{0}=3 / 4, \rho_{i}^{0}=1 / 3$ and $\Omega=\emptyset$. (b)-(d) Approximated domains $\Omega_{d}, d=1,2,3$, superimposed to the TD computed for $\Omega=\Omega_{d}$ and the corresponding updated values of the thermal parameters $\kappa_{i}$ and $\rho_{i}$.

proved we perform further gradient iterations to update the parameters and so on. The algorithm stops if any of the following stopping criteria is satisfied:

- $\operatorname{meas}\left(\Omega_{d} \backslash \Omega_{d-1}\right)$ is small,

- $\left|\kappa_{i}^{q}-\kappa_{i}^{q-1}\right|+\left|\rho_{i}^{q}-\rho_{i}^{q-1}\right|$ is small and $\left\|U_{\text {meas }}-U_{\text {total }}\right\|$ is small,

- $J\left(\mathbb{R}_{-}^{2} \backslash \Omega_{d}, \kappa_{i}^{q}, \rho_{i}^{q}\right)$ is small.

\section{A numerical example}

In this section we present a numerical example to illustrate the feasibility of our reconstruction algorithm. We consider a simple geometry where $\Omega$ is the ellipse $\Omega=\left\{(x, y) \in \mathbb{R}^{2}, x^{2} / 0.55^{2}+(y+1)^{2} / 0.35^{2}<1\right\}$, with thermal parameters $\kappa_{i}=$ $1 / 2$ and $\rho_{i}=1$. In the exterior medium the values of the parameters are $\kappa_{e}=1$ and $\rho_{e}=1 / 5$.

Synthetic data are created solving (2-4) by means of the Laplace transform with respect to time and a boundary element formulation in space (see $[4,12]$ for details). A relative $1 \%$ Gaussian error was added at each observation point to both avoid inverse crimes and to simulate measurement errors. We have considered six incident waves of the form (1) generated at the uniformly distributed source points represented in all the plots in figure 2 by ' $\bullet$ ' marks. Measurements of the temperature were taken at the seven observation points represented by ' $\times$ ' marks at 10 uniformly distributed times in the time interval $[0.05,0.5]$.

We started the algorithm by choosing the initial values $\kappa_{i}^{0}=3 / 4$ and $\rho_{i}^{0}=1 / 3$. To obtain an initial guess for the domain, we computed the TD in the sampling region $[-3,3] \times[-2.5,0]$. This yields the colormap represented in figure 2(a). Dark 
(a)

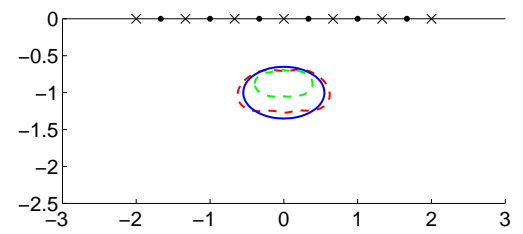

(b)

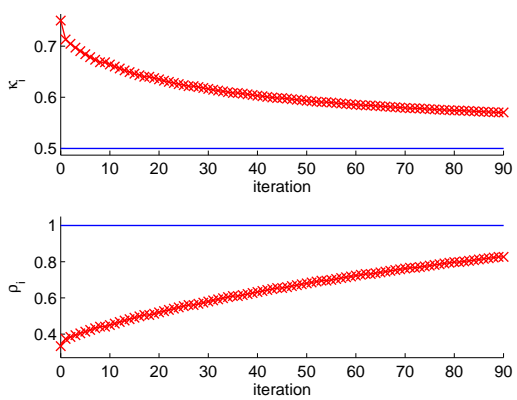

Fig. 3 Final reconstrucion after 10 iterations with respect to the domain. (a) Initial (green dashed line), predicted (red dashed line) and true (blue solid line) objects. (b) Values of the thermal parameters $\kappa_{i}$ and $\rho_{i}$ versus the number of iterations.

blue colors indicate the regions where the TD takes large negative values, at which the objects should be located. The boundary of the true defect is represented by a solid white line in all plots in figure 2 . Our initial guess $\Omega_{1}$ is represented in figure 2(b). We set now $\Omega=\Omega_{1}$ and perform eight iterations with the gradient method to correct the values of $\kappa_{i}$ and $\rho_{i}$. In the next step, the values of the parameters are fixed and the TD is again computed. In figure 2(c) we represent the updated object $\Omega_{2}$. The hybrid algorithm alternates eight iterations with the gradient method with one TD computation. It stopped at the tenth iteration with respect to the domain. The first three approximated domains are represented in figure 2. In figure 3(a) we show the true object (solid blue line), the initial guess $\Omega_{1}$ (dashed green line), and the final reconstruction $\Omega_{10}$ (dashed red line). The values of $\kappa_{i}$ and $\rho_{i}$ throughout the iterations are given in figure 3(b). Two identical values mark a TD computation to update the domain. The final approximations were $\kappa_{i}^{\text {final }}=0.5703$ (recall that the true value is 0.5 ), and $\rho_{i}^{\text {final }}=0.8266$ (while the true value is 1 ). We have obtained a satisfactory reconstruction taking into account that no a priori knowledge about the number, size or location of the objects is assumed, and that few data were available.

In our example, we found a sequence of enlarging sets, i.e., satisfying $\Omega_{d} \subset$ $\Omega_{d+1}$. An example where the TD provides a sequence of defects where $\Omega_{d} \nsubseteq \Omega_{d+1}$ for some values of $d \in \mathbb{N}$ can be found in [6]. The interested reader may find some reconstructions with other geometries, multiple objects and different weight functions $f(t)$ in $[4,6]$. Furthermore, a gallery of comparisons varying the different parameters of the problem, namely, the number of source points and/or observation points, the number of time observations, etc, can be found in [4] for a simplified situation where the interior parameters are assumed to be known and in [6] for a related problem with unknown domains and parameters.

Acknowledgements The authors are partially supported by the Spanish Government research project TRA2010-18054 and the Spanish Ministerio de Economía y Competitividad Grants No. FIS2011-222888-C02-02 and FIS2010-22438-E. 


\section{References}

1. Bonnet, M., Constantinescu, A.: Inverse problems in elasticity. Inverse Problems, vol. 21, pp. $1-50$ (2005)

2. Carpio, A., Rapún, M.-L.: Topological derivatives for shape reconstruction, In: Inverse Problems and Imaging, Lecture Notes in Mathematics, pp. 85-134. Springer (2008)

3. Carpio, A., Rapún, M.-L.: Solving inhomogenous inverse problems by topological derivative methods. Inverse Problems vol. 24, art. num. 045014 (2008)

4. Carpio, A., Rapún, M.-L.: Domain reconstruction using photothermal techniques. J. Comput. Phys. vol. 15, pp. 8083-8106 (2008)

5. Carpio, A. Rapún, M.-L.: Hybrid topological derivative and gradient based methods for nondestructive testing. Abstract and Applied Analysis 2013, art. num. 816134 (2013)

6. Carpio, A., Rapún, M.-L.: Parameter identification in photothermal imaging. J. Math. Imaging Vis. vol. 49, pp. 273-288 (2014)

7. Feijoo, G.R.: A new method in inverse scattering based on the topological derivative. Inverse Problems, vol. 20, pp. 1819-1840 (2004)

8. Garreau, S., Guillaume, P., Masmoudi, M.: The topological asymptotic for PDE sistems: the elasticity case. SIAM J. Control Optim., vol. 39, pp. 1756-1778 (2001)

9. Garrido, F., Salazar, A.: Thermal wave scattering by spheres. J. Appl. Phys. vol. 95, pp. 140-149 (2004)

10. Guzina, B.B., Bonnet, M.: Small-inclusion asymptotics of misfit functionals for inverse problems in acoustics. Inverse Problems, vol. 22, pp. 1761-1785 (2006)

11. Hettlich, F.: Fréchet derivatives in inverse scattering problems. Inverse Problems vol. 11, pp. 371-382 (1995)

12. Hohage, T., Sayas, F.-J.: Numerical approximation of a heat diffusion problem by boundary element methods using the Laplace transform. Numer. Math. vol. 102, pp. 67-92 (2005)

13. Kirsch, A.: The domain derivative and two applications in inverse scattering theory. Inverse Problems, vol. 9, pp. 81-96 (1993)

14. Laliena, A., Sayas, F.-J.: LDBEM in diffusion problems. In: Proceedings of XIX CEDYA/IX CMA (electronic version) (2005)

15. Laurain, A., Hintermüller, M., Freiberger, M., Scharfetter, H.: Topological sensitivity analysis in fluorescence optical tomography. Inverse Problems, vol. 29, art. num. 025003 (2013)

16. Litman, A., Lesselier, D., Santosa, F.: Reconstruction of a two-dimensional binary obstacle by controlled evolution of a level-set. Inverse Problems, vol. 14, pp. 685-706 (1998)

17. López-Fernández, M., Palencia, C.: On the numerical inversion of the Laplace transform of ertain holomorphic mappings. Appl. Numer. Math. vol. 51, pp. 289-303 (2004)

18. Mendioroz, A., Castelo, A., Celorrio, R., Salazar, A.: Characterization of vertical buried defects using lock-in vibrothermography: I Direct problem. Meas. Sci. Technol. vol. 24, art. num. 065601 (2013)

19. Potthast, R.: Fréchet differentiability of the solution to the acoustic Neumann scattering problem with respect to the domain. Journal of Inverse and Ill-Possed Problems, vol. 4, pp. 67-84 (1996)

20. Samet, B., Amstutz, S., Masmoudi, M.: The topological asymptotic for the Helmholtz equation. SIAM J. Control Optim. vol. 42, pp. 1523-1544 (2003)

21. Santosa, F.: A level-set approach for inverse problems involving obstacles. Optimisation et Calcul des Variations, vol. 1, pp. 17-33 (1996)

22. Sokolowski, J., Zolésio, J.P.: Introduction to shape optimization. Shape Sensitivity Analysis. Springer, Heidelberg (1992). 